\begin{tabular}{cc} 
Sharif University of Technology \\
Scientia Iranica \\
SCIENTIA & Transactions B: Mechanical Engineering \\
I RAN I CA & \\
\hline
\end{tabular}

\title{
Rheology of Burgers' model with Cattaneo-Christov heat flux in the presence of heat source/sink and magnetic field
}

\author{
Aqsa $^{a}$, M.Y. Malik ${ }^{a}$, A. Imtiaz ${ }^{a}$, and M. Awais ${ }^{b, *}$ \\ a. Department of Mathematics, Quaid-i-Azam University, Islamabad, 44000, Pakistan. \\ b. Department of Mathematics, COMSATS Institute of Information Technology, Attock, 43600, Pakistan. \\ Received 7 December 2016; received in revised form 20 August 2017; accepted 27 January 2018
}

\section{KEYWORDS}

Burgers model;

Cattaneo-Christov

heat flux;

Streamlines;

Magnetohydrodynamics.

\begin{abstract}
This study presents the characteristics of Cattaneo-Christov heat flux model for the boundary layer flow of Burgers' fluid model. Instead of simple Fourier's law of heat conduction, we presented the Cattaneo-Christov model to analyze the thermal relaxation properties when the heat source/sink was present in the system. Mathematical modeling of the laws of momentum and energy were presented under the order analysis approach. It was revealed that the term " $\sigma B_{0}^{2} u / \rho$ " was for the hydro-magnetic rheology of the Newtonian model whereas the generalized magnetic field term was for the Burgers' model, which was incorporated in the current analysis. Suitable transformations were utilized for the conversion of partial differential system into coupled nonlinear set of ordinary differential equations, which was tackled analytically through homotopy analysis technique. The plots of various physical quantities are presented, showing the dynamics of the considered analysis. Streamlines for Burgers' and Newtonian models are presented, which show their difference in rheology. Numerical values for skin friction and surface heat transfer rate are presented in tables.
\end{abstract}

(C) 2019 Sharif University of Technology. All rights reserved.

\section{Introduction}

Heat transfer dynamics has been one of the hot topics in the present years due to its ample applications in several engineering and technical processes, e.g., nuclear reactors, energy generators, biological and medical appliances, electronic device cooling, heat pumps, etc.

The famous mathematician and physicist Fourier presented a law of heat conduction, which is termed the Fourier law of heat conduction, in 19th century to explore the properties of heat transfer under various

\footnotetext{
*. Corresponding author.

E-mail address: awais_mm@yahoo.com (M. Awais)
}

configurations and technical aspects. Researchers have utilized the Fourier's law to investigate several industrial and engineering problems and have found that there are some disadvantages with this model. For instance, this model results in parabolic differential equation, which specifies that the initial disturbance should be experienced by the material medium immediately. To overcome this difficulty, Cattaneo [1] presented a modification to the Fourier's law by introducing a relaxation time parameter. Christov [2] presented the frame-indifferent mathematical modelling and generalization of the Cattaneo's model through applying convected derivative. Later on, several researchers and mathematicians extended this concept under various flow aspects. For instance, Tibullo and Zampoli [3] analyzed uniqueness result for the Cattaneo-Christov 
heat conduction law for incompressible fluids. They performed a validity analysis for the Cattaneo-Christov heat law and established a uniqueness of solutions for the boundary initial value problems for the incompressible fluid flows. Thermal instability in Brinkman porous media with Cattaneo-Christov heat flux was presented by Haddad [4]. He concluded that by employing the Cattaneo-Christov theory, one could achieve significant and pronounced effects in finding the convection instability threshold. Coupled flow and heat transfer in a viscoelastic fluid with CattaneoChristov heat flux model was presented by Han et al. [5]. They considered the upper-convected Maxwell fluid flow with slip boundary conditions and employed homotopy analysis method for computations. They also presented a comparison of results for Fourier law and Cattaneo-Christov heat flux model. Hayat et al. [6] presented hydromagnetic flow of an Oldroyd-B fluid with homogeneous-heterogeneous reactions. They considered the Cattaneo-Christov heat fluid model and computed the solutions by employing homotopy analysis method. Ali and Sandeep [7] analyzed the CattaneoChristov model for radiative heat transfer of magnetohydrodynamic Casson-ferrofluid. They presented the numerical study. Kumar et al. [8] investigated the magneto hydrodynamic Cattaneo-Christov flow past a cone and a wedge with variable heat source/sink. Free convective MHD Cattaneo-Christov flow over three different geometries with thermophoresis and Brownian motion was analyzed by Babu et al. [9]. Cross diffusion effects on MHD flow over three different geometries with Cattaneo-Christov heat flux were studied by Reddy et al. [10]. Zhao et al. [11] presented the unsteady Marangoni convection heat transfer of fractional Maxwell fluid with Cattaneo heat flux. Sui et al. [12] analyzed the boundary layer heat and mass transfer with Cattaneo-Christov double-diffusion in upper-convected Maxwell nanofluid past a stretching sheet with slip velocity. MHD viscoelastic flow and heat transfer over a vertical stretching sheet with Cattaneo-Christov heat flux effects were investigated by Li et al. [13].

In the present analysis, we have discussed the Cattaneo-Christov heat flux model for the Burgers' fluid model when heat source/sink is present in the system. Mathematical modeling of the laws of momentum and energy is presented under the order analysis approach. Suitable transformations are utilized for the conversion of partial differential system into coupled nonlinear set of ordinary differential equations, which is tackled analytically through homotopy analysis technique. Note that with the advent of computers, the approximate solutions in fluid dynamics have lost their importance to some extent as more and better numerical algorithms have been developed to numerically solve the increasingly realistic, but more complicated problems. Nevertheless, approximate analytical solutions still have their relevance for the following reasons: Firstly, they give the solution for each point within the domain of interest. Secondly, a nicely produced approximate solution, requiring a minimal effort and having a reasonable amount of accuracy, is always handy for an engineer, scientist, or applied mathematician, who can obtain a solution quickly, thereby gaining a valuable insight into the essentials of the problem. Thirdly, even with most of the scientific packages, some initial guess is required for the solution, as the algorithms, in general, are not globally convergent. In such situations, approximate solutions can provide an excellent starting guess that can be readily refined. Keeping the above facts in mind, we develop series solution by employing a Homotopy Analysis Method (HAM) [14-25]. The plots of various physical quantities are presented showing the dynamics of the considered analysis. Streamlines for Burgers' and Newtonian models are presented, which show their difference in rheology. Numerical values for skin friction and surface heat transfer rate are presented in the tables.

\section{Mathematical formulation}

Let us consider the dynamics of Burgers' fluid past a conducting wall in the region of stagnation point. The conducting wall is located along $x$-axis and an incompressible Burgers' fluid fills the space $y>0$ as shown in Figure 1. A uniform magnetic field $\mathbf{B}=\left\{0, B_{0}, 0\right\}$ is applied along $y$-axis and the effects of induced magnetic field are neglected under the assumptions of small magnetic Reynolds' number. A conducting wall undergoes stretching phenomenon with velocity $U_{s}(x)$ and the free stream velocity is taken as $U_{e}(x)$. The boundary layer equations governing the flow in the present flow situation are of the form [28]:

$$
\frac{\partial u}{\partial x}+\frac{\partial v}{\partial y}=0
$$

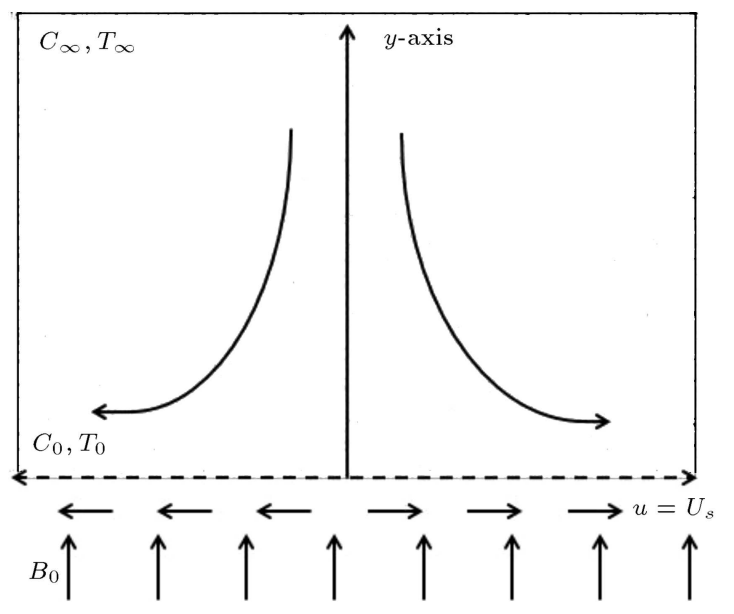

Figure 1. Geometry of the problem. 


$$
\begin{aligned}
& u \frac{\partial u}{\partial x}+v \frac{\partial u}{\partial y}+\lambda_{1}\left(u^{2} \frac{\partial^{2} u}{\partial x^{2}}+v^{2} \frac{\partial^{2} u}{\partial y^{2}}+2 u v \frac{\partial^{2} u}{\partial x \partial y}\right) \\
& +\lambda_{2}\left(u^{3} \frac{\partial^{3} u}{\partial x^{3}}+v^{3} \frac{\partial^{3} u}{\partial y^{3}}\right. \\
& +u^{2}\left(\frac{\partial^{2} u}{\partial x^{2}} \frac{\partial u}{\partial x}-\frac{\partial u}{\partial y} \frac{\partial^{2} v}{\partial x^{2}}+2 \frac{\partial v}{\partial x} \frac{\partial^{2} u}{\partial x \partial y}\right) \\
& +3 v^{2}\left(\frac{\partial v}{\partial y} \frac{\partial^{2} u}{\partial y^{2}}+\frac{\partial u}{\partial y} \frac{\partial^{2} u}{\partial x \partial y}\right) \\
& +3 u v\left(u \frac{\partial^{3} u}{\partial x^{2} \partial y}+v \frac{\partial^{3} u}{\partial x \partial y^{2}}\right)+2 u v\left(\frac{\partial u}{\partial y} \frac{\partial^{2} u}{\partial x^{2}}\right. \\
& \left.\left.+\frac{\partial v}{\partial x} \frac{\partial^{2} u}{\partial y^{2}}+\frac{\partial v}{\partial y} \frac{\partial^{2} u}{\partial x \partial y}-\frac{\partial u}{\partial y} \frac{\partial^{2} v}{\partial x \partial y}\right)\right)=\nu\left\{\frac{\partial^{2} u}{\partial y^{2}}\right. \\
& \left.+\lambda_{3}\left(u \frac{\partial^{3} u}{\partial x \partial y^{2}}+v \frac{\partial^{3} u}{\partial y^{3}}-\frac{\partial u}{\partial x} \frac{\partial^{2} u}{\partial y^{2}}-\frac{\partial u}{\partial y} \frac{\partial^{2} v}{\partial y^{2}}\right)\right\} \\
& -\frac{\sigma B_{0}^{2}}{\rho}\left(u-U_{e}+\lambda_{1} v \frac{\partial u}{\partial y}\right)+U_{e} \frac{d u}{d x} \\
& -\frac{\sigma B_{0}^{2} \lambda_{2}}{\rho}\left(u \frac{\partial v}{\partial x} \frac{\partial u}{\partial y}-v \frac{\partial u}{\partial x} \frac{\partial u}{\partial y}+u v \frac{\partial^{2} u}{\partial x \partial y}+v^{2} \frac{\partial^{2} u}{\partial y^{2}}\right) \\
& (2)
\end{aligned}
$$

corresponding to the wall conditions:

$$
\begin{array}{ll}
u=U_{s}, & \text { at } \quad y=0, \\
u=U_{e} & \text { as } y \rightarrow \infty .
\end{array}
$$

In the above differential system, $u$ and $v$ represent the velocity components along $x$ - and $y$-directions, respectively, $\lambda_{1}$ and $\lambda_{2}$ represent the relaxation times, and $\lambda_{3}$ corresponds to retardation time. We would like to mention here that for $\lambda_{3}=0$, the results for an Oldroyd-B fluid model can be deduced and for $\lambda_{2}=\lambda_{3}=0$, one can obtain the results for a Maxwell fluid model. Also, the results for Newtonian fluid model can be deduced for $\lambda_{1}=\lambda_{2}=\lambda_{3}=0$. Moreover, $\rho$ represents the density of the fluid and $\sigma$ represents the electrical conductivity.

The Cattaneo-Christov heat flux model can be written as $[1,2]$ :

$$
q+\lambda\left(\frac{\partial q}{\partial t}+V \cdot \nabla q-q . \nabla V+(V \cdot \nabla) q\right)=-k \nabla T
$$

where $q$ represents the heat flux, $\lambda$ the heat flux relaxation time, $T$ the temperature of the fluid, $k$ the thermal conductivity, and $V$ the velocity vector. The above equation corresponds to Fourier's law when $\lambda=0$.
The energy equation in the presence of internal heat generation/absorption effects takes the following form $[1,2]$ :

$$
\rho c_{p} V \cdot \nabla T=-\nabla \cdot q+Q\left(T-T_{\infty}\right) .
$$

Making use of Eq. (4) into Eq. (5), we get the following form of energy equation:

$$
\begin{aligned}
u \frac{\partial T}{\partial x} & +v \frac{\partial T}{\partial y}+\lambda\left(u^{2} \frac{\partial^{2} T}{\partial x^{2}}+v^{2} \frac{\partial^{2} T}{\partial y^{2}}+2 u v \frac{\partial^{2} T}{\partial x \partial y}\right. \\
& \left.+u \frac{\partial u}{\partial x} \frac{\partial T}{\partial x}+u \frac{\partial v}{\partial x} \frac{\partial T}{\partial y}+v \frac{\partial v}{\partial y} \frac{\partial T}{\partial y}\right) \\
& -\frac{Q\left(T-T_{\infty}\right)}{\rho c_{p}}+\frac{Q}{\rho c_{p}} \lambda\left(u \frac{\partial T}{\partial x}+v \frac{\partial T}{\partial y}\right) \\
& =\frac{k}{\rho c_{p}}\left(\frac{\partial T^{2}}{\partial x^{2}}+\frac{\partial T^{2}}{\partial y^{2}}\right) .
\end{aligned}
$$

The associated boundary conditions for the fluid's temperature are:

$$
\begin{aligned}
& T=T_{w} \quad \text { at } \quad y=0, \\
& T \rightarrow T_{\infty} \quad \text { as } \quad y \rightarrow \infty,
\end{aligned}
$$

where $Q$ is the internal heat generation/absorption coefficient, $c_{p}$ is specific heat constant, $T_{w}$ is the wall temperature, and $T_{\infty}$ is the ambient temperature.

We proceed by taking the following stream function:

$$
u=\frac{\partial \psi}{\partial y} \quad \text { and } \quad v=-\frac{\partial \psi}{\partial x},
$$

where:

$$
\psi=\sqrt{c \nu} x f(\eta), \quad \theta(\eta)=\frac{T-T_{\infty}}{T_{w}-T_{\infty}}, \quad \eta=\sqrt{\frac{c}{\nu} y}
$$

Making use of the above variables, Eq. (1) is automatically satisfied and from Eqs. (2) and (6), one arrives at the following expressions:

$$
\begin{aligned}
& f^{\prime \prime \prime}-f^{\prime 2}+f f^{\prime \prime}+\beta_{1}\left(2 f f^{\prime} f^{\prime \prime}-f^{2} f^{\prime \prime \prime}\right) \\
& \quad+\beta_{2}\left(f^{3} f^{\prime \prime \prime \prime}-2 f f^{\prime 2} f^{\prime \prime}-3 f^{2} f^{\prime \prime 2}\right) \\
& \quad+\beta_{3}\left(f^{\prime \prime 2}-f f^{\prime \prime \prime \prime}\right)-M^{2}\left(f^{\prime}-\beta_{1} f f^{\prime \prime}+\beta_{2} f^{2} f^{\prime \prime \prime}\right) \\
& \quad+A^{2}+M^{2} A=0 \\
& \theta^{\prime \prime}+\operatorname{Pr}\left(f \theta^{\prime}+h_{s} \theta+\gamma\left(f^{2} \theta^{\prime \prime}+f f^{\prime} \theta^{\prime}-h_{s} f \theta^{\prime}\right)\right)=0 .
\end{aligned}
$$


Along with associated wall conditions:

$$
\begin{aligned}
& f^{\prime}(\eta)=1, \quad f(\eta)=0, \quad \theta(\eta)=1 \quad \text { at } \quad \eta=0, \\
& f^{\prime}(\eta)=A, \quad \theta(\eta)=0 \quad \text { as } \quad \eta \rightarrow \infty, \quad(1)
\end{aligned}
$$

where Deborah numbers $\beta_{1}$ and $\beta_{3}$ are in terms of relaxation times, and the Deborah number $\beta_{2}$ is in terms of retardation times. Furthermore, $\operatorname{Pr}$ is the Prandtl number, $h_{s}$ is the heat transfer parameter, $\gamma$ is the conjugate thermal relaxation parameter, $M$ is the Hartman number, and $A$ is the ratio of stretching velocity to free stream velocity. They are defined as:

$$
\begin{array}{lll}
\beta_{1}=c \lambda_{1}, & \beta_{2}=c^{2} \lambda_{2}, & \beta_{3}=c \lambda_{3}, \\
\operatorname{Pr}=\frac{\nu}{\alpha}, & M^{2}=\frac{\sigma B_{0}^{2}}{\rho c}, & A=\frac{a}{c} .
\end{array}
$$

\section{Results and discussion}

This section aims to analyze the effects of various physical quantities including Deborah numbers, stagnation point, thermal relaxation parameter, heat source/sink, Prandtl number, etc. on the velocity and temperature distribution profiles. Therefore, we have prepared Figures 2-10 and Tables 1-3. Figures 2 and 3 portray the streamlines for the Newtonian and Burgers' fluid models. It is noted that the streamlines for Burgers' fluid model are quite different from the streamlines for Newtonian fluid model. It is because of the presence of various relaxation and retardation times in the stress tensor of Burgers' model. Figure 4 presents the variation of stagnation point parameter $A$ on the velocity field. It is noted that with increase in $A$, the velocity increases and the momentum boundary layer deceases in the region $0.0<A<1.0$, whereas for

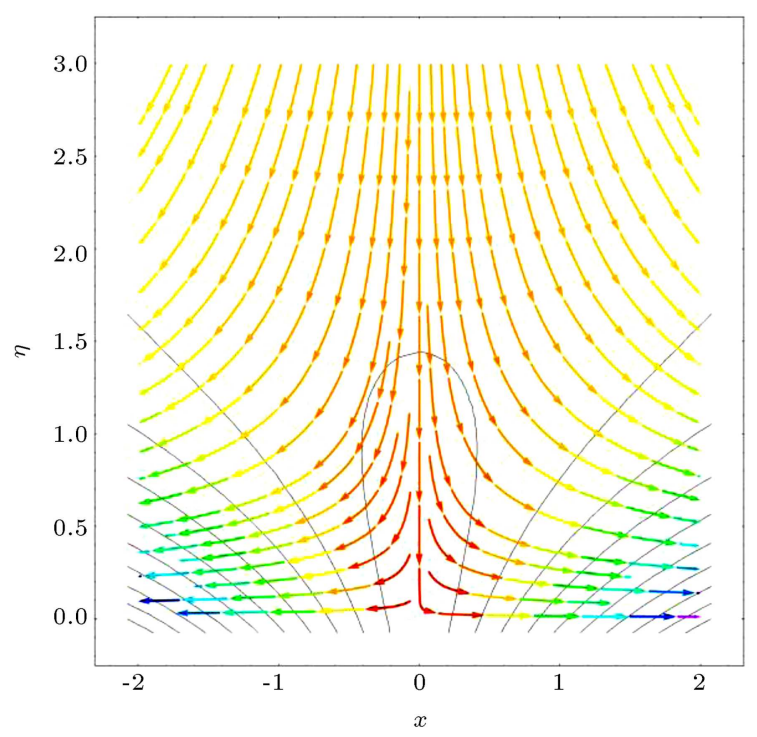

Figure 2. Rheology of Newtonian model.

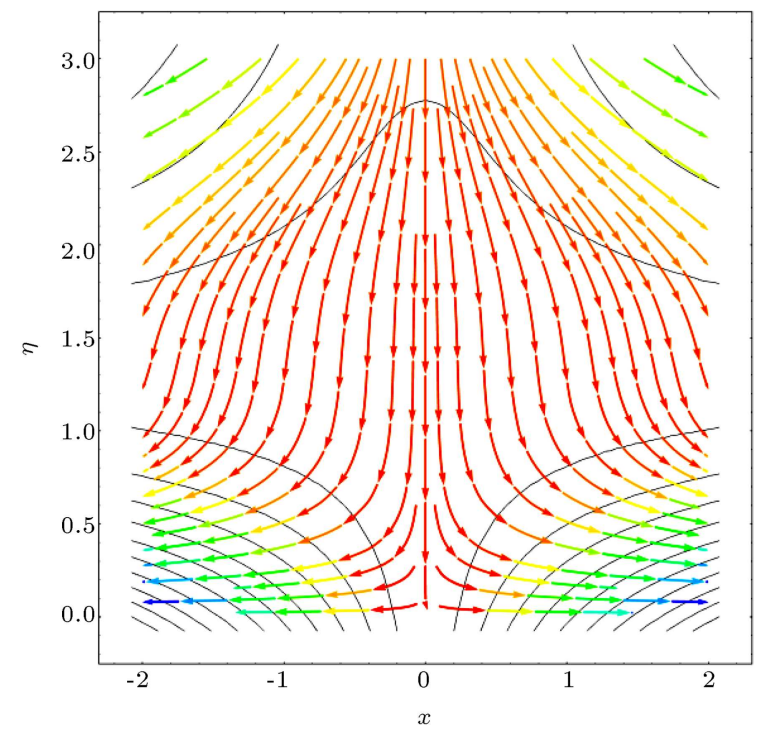

Figure 3. Rheology of Burgers' model.

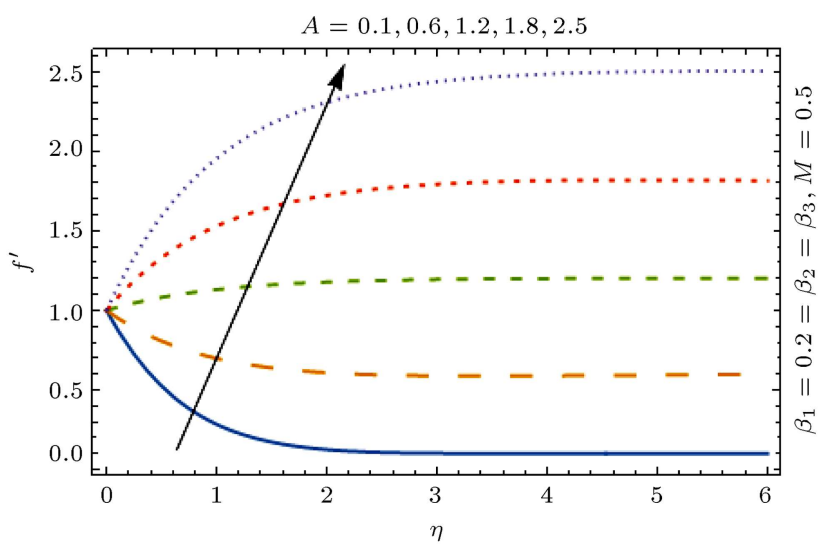

Figure 4. Effects of $A$ on velocity.

$A>1.0$, the momentum boundary layer also increases. Thus, we can conclude that the larger values of $A$ accompany the higher free stream velocities, which enhance velocity and momentum boundary layer of the fluid. Moreover, for $A=1.0$, both velocity and momentum boundary layer vanish. Therefore, we can conclude that $A=1.0$ is a critical value at which the behavior of the momentum boundary layer changes. Figure 5 presents a comparison of Newtonian and Burgers' fluid models. It is observed that the magnitudes of velocity profile and momentum boundary layer are smaller in the rheology of Burgers model than in the rheology of Newtonian model. From physical point of view, we can say that extra physical quantities $\left(\beta_{1}, \beta_{2}\right.$, and $\beta_{3}$ ) are non-zero for Burgers' fluid model. Positive values of $\beta_{1}, \beta_{2}$, and $\beta_{3}$ correspond to viscous as well as elastic effects, which retard the flow, and, hence, the boundary layer will be thinner. Figure 6 presents the effects of magnetic field on the flow profile. It is noted that velocity profile decreases with increase in magnetic field parameter $M$. From physical point of 


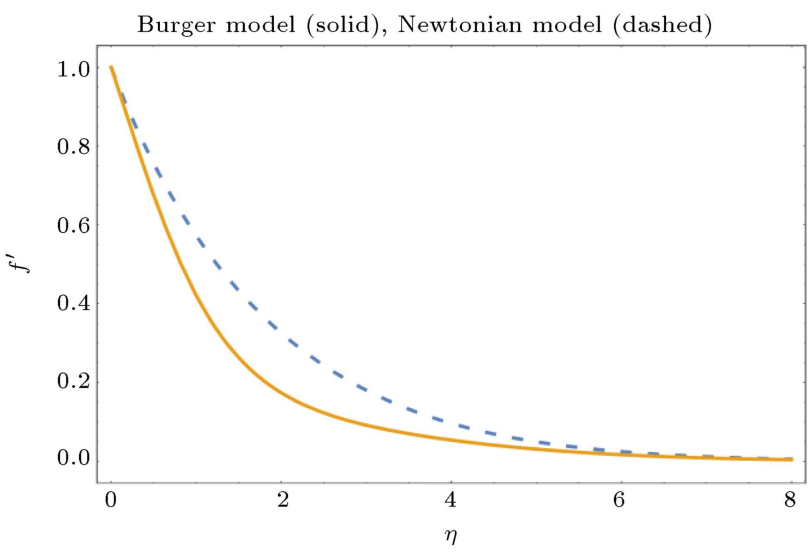

Figure 5. Newtonian versus Burgers' models.

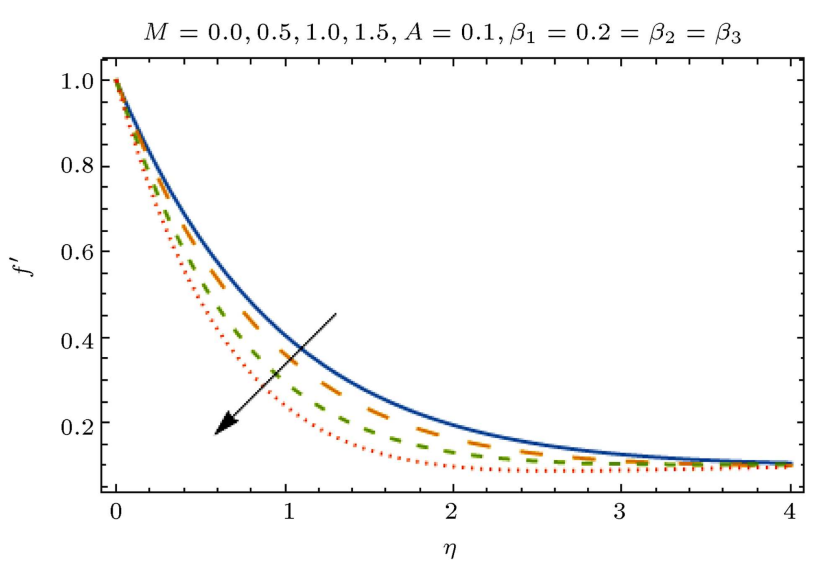

Figure 6. Effects of $M$ on velocity.

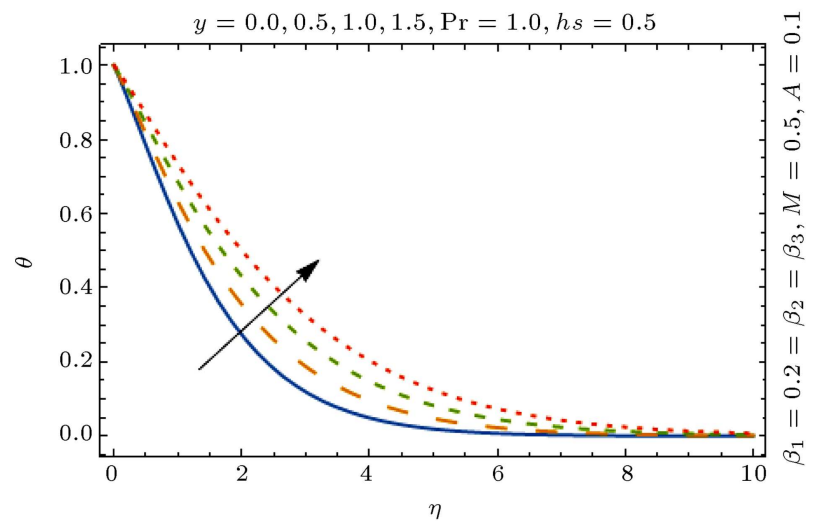

Figure 7. Effects of $\gamma$ on temperature.

view, we can say that the apparent viscosity increases when magnetic field is applied to any fluid, which enhances the ability of the fluid to transmit the force in a precisely controlled manner, giving rise to many possible rheostat-based applications, including power generation, electromagnetic casting of metals, etc. Figure 7 elucidates the effects of thermal relaxation time of the temperature profile. It is noted that the temperature profile has an inverse relationship with the thermal relaxation time. We see that the temperature

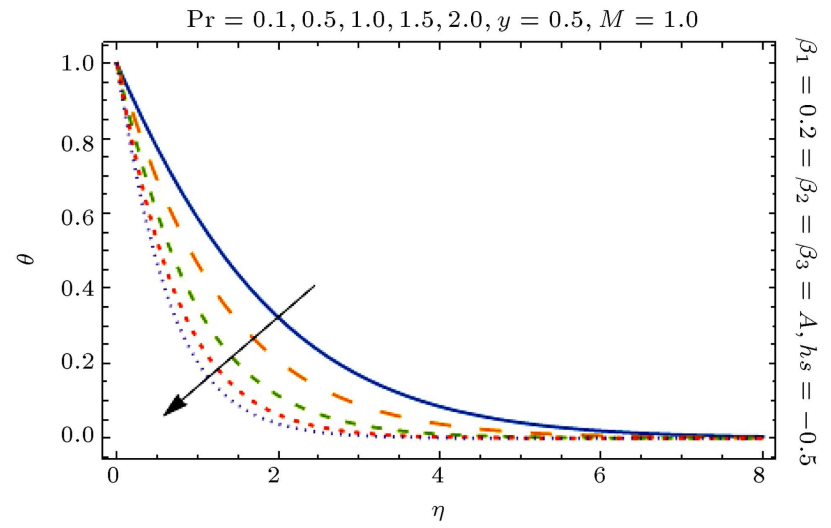

Figure 8. Effects of Pr on temperature.

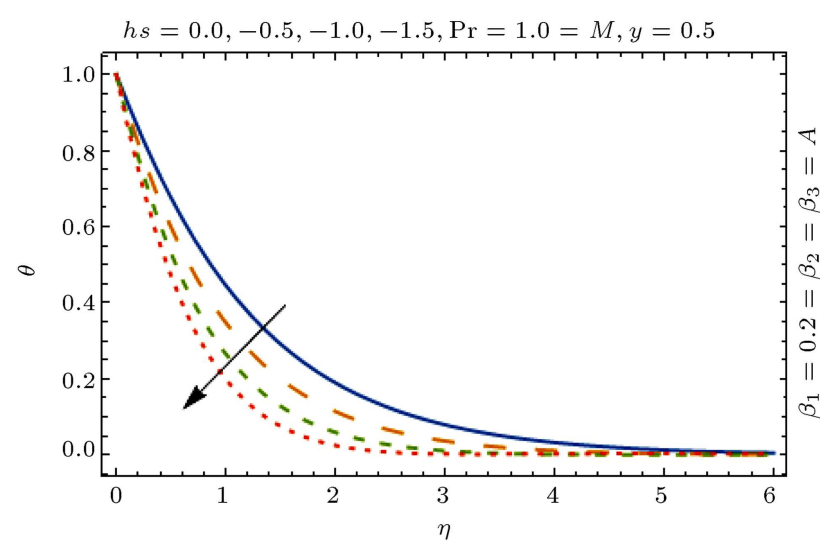

Figure 9. Effects of heat sink on temperature.

profile approaches free stream quite instantly with larger values of thermal relaxation parameter. Figure 8 presents the effects of Prandtl number, $\mathrm{Pr}$, on the temperature profile. Reduction in temperature profile and thermal boundary layer is noted when Prandtl number is increased. It is quite physical, since the Prandtl number is the ratio of momentum diffusivity to thermal diffusivity and the thermal diffusivity is weaker for larger values of Prandtl number due to decrease in thermal diffusion rate. Note that reduction in thermal diffusion results in decrease in temperature. The effects of internal heat generation/absorption parameters $h_{s}$ on the temperature profile are presented in Figures 9 and 10 . It is noted that the temperature profile, $\theta(\eta)$, decreases when the heat sink is present in the system, whereas for the case of heat source, the temperature profile increases. From physical point of view, we can conclude that the presence of heat source/sink in the system plays vital role in terms of controlling temperature of the liquids. This is very important from an industrial point of view, since the quality of final product strongly depends upon the heat transfer rate and its value. Table 1 presents a comparative study of the obtained results with the already available literature. It is noted that the present results in a limiting case are in good agreement with the published 


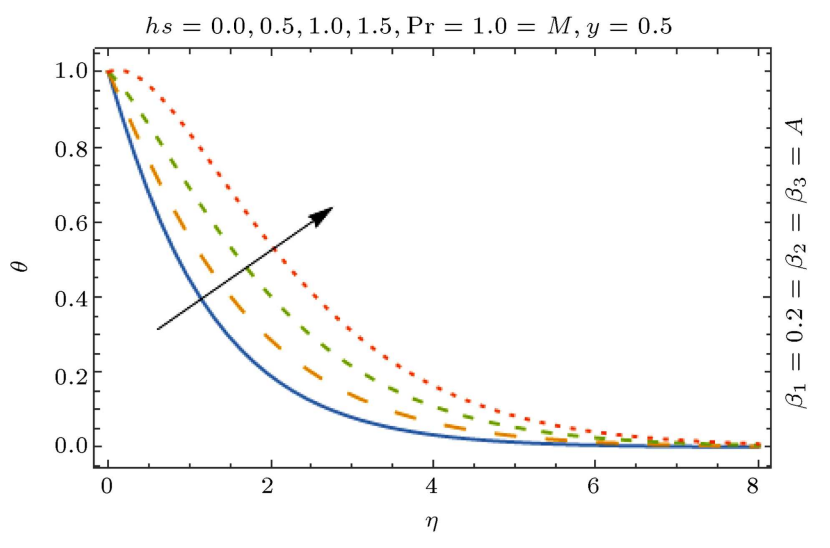

Figure 10. Effects of heat source on temperature.

Table 1. Comparison of $f^{\prime \prime}(0)$ with published results when $A=0.0=\beta_{2}=\beta_{3}=M$.

\begin{tabular}{cccc}
\hline $\boldsymbol{\beta}_{\mathbf{1}}$ & $\begin{array}{c}\text { Our } \\
\text { results }\end{array}$ & $\begin{array}{c}\text { Sadghey } \\
\text { et al. [26] }\end{array}$ & $\begin{array}{c}\text { Mukhopadhyay } \\
{[\mathbf{2 7}]}\end{array}$ \\
\hline 0.0 & 1.000000 & 1.00000 & 0.999999 \\
0.4 & 1.101904 & 1.10084 & 1.101851 \\
0.8 & 1.196712 & 1.19872 & 1.196693 \\
\hline
\end{tabular}

Table 2. Behavior of $f^{\prime \prime}(0)$ with different values of Burgers parameter $\beta_{2}$.

\begin{tabular}{ll}
\hline $\boldsymbol{\beta}_{\mathbf{2}}$ & $-\boldsymbol{f}^{\prime \prime}(\mathbf{0})$ \\
\hline 0.0 & 0.96652 \\
0.2 & 0.97951 \\
0.4 & 0.99328 \\
0.6 & 1.00773 \\
0.8 & 1.01582 \\
1.0 & 1.05219 \\
\hline
\end{tabular}

results. Table 2 is prepared to analyze the behavior of $f^{\prime \prime}(0)$ for different values of Burgers parameter, $\beta_{2}$. It is noted that the numerical values of skin friction, $f^{\prime \prime}(0)$ increase with larger values of Burgers parameter, $\beta_{2}$. The numerical values of heat transfer rate at the wall $\theta^{\prime}(0)$ for different physical parameters are presented in Table 3. It is noted that the magnitude of $\theta^{\prime}(0)$ decreases with Burgers parameter, thermal relaxation time, and internal heat source/sink quantity.

\section{Concluding remarks}

We discussed the Cattaneo-Christov heat flux model for the Burgers fluid model when the heat source/sink was present in the system. The important observations are as follows:

- Velocity profile and momentum boundary layer for Burger model are quite lower due to extra viscoelastic effects;
Table 3. Numerical values of $\theta^{\prime}(0)$ with different physical parameters.

\begin{tabular}{cccc}
\hline $\boldsymbol{\beta}_{\mathbf{2}}$ & $\boldsymbol{\gamma}$ & $\boldsymbol{h}_{\boldsymbol{s}}$ & $\boldsymbol{- \boldsymbol { \theta } ^ { \prime } ( \mathbf { 0 } )}$ \\
\hline 0.0 & 0.3 & -0.2 & 0.76621 \\
0.2 & & & 0.75992 \\
0.4 & & & 0.74165 \\
0.2 & 0.0 & & 0.76105 \\
& 0.3 & & 0.75992 \\
& 0.6 & & 0.74319 \\
& 0.3 & -0.2 & 0.75992 \\
& & 0.0 & 0.60445 \\
& & 0.2 & 0.41284 \\
\hline
\end{tabular}

- Streamlines for Newtonian and Burgers' models show their difference in rheology;

- Temperature profile and thermal boundary layer are quite low in the Cattaneo-Christov heat flux model when compared with the basic Fourier's law;

- Reduction in temperature profile and thermal boundary layer is noted with larger Prandtl number values;

- Presence of heat source/sink in the system can efficiently control the temperature to the desired value.

\section{Acknowledgement}

The authors are grateful to the editor and the referees for their constructive comments to improve the manuscript.

\section{References}

1. Cattaneo, C. "Sulla Conduzione del calore", Atti Semin. Mat. Fis. Univ. Modea Reggio Emilia, 3, pp. 83-101 (1948).

2. Christov, C.I. "On frame indifferent formulation of Maxwell Cattaneo model of finite speed heat conduction", Mech. Res. Comm., 36(4), pp. 481-486 (2009).

3. Tibullo, V. and Zampoli, V. "A uniquemess result for the Cattaneo-Christov heat conduction model applied to incompressible fluids", Mech. Res. Comm., 38(1), pp. 77-79 (2011).

4. Haddad, S.A.M. "Thermal instability in Brinkman porous media with Cattaneo-Christov heat flux", Int. J. Heat Mass Trans., 68, pp. 659-668 (2014).

5. Han, S., Zheng, L., Li, C., and Zhang, X. "Coupled flow and heat transfer in viscoelastic fluids with Cattaneo-Christov heat flux model", Appl. Math. Lett., 38, pp. 87-93 (2014).

6. Hayat, T., Imtiaz, M., Alsaedi, A., and Almezal, S. "On Cattaneo-Christov heat flux in MHD flow of Oldroyd-B fluid with homogeneous-heterogeneous 
reactions", J. Mag. Mag. Mat., 401, pp. 296-303 (2016).

7. Ali, M.E. and Sandeep, N. "Cattaneo-Christov model for radiative heat transfer of magnetohydrodynamic Casson-ferrofluid: A numerical study", Results Physics, 7, pp. 21-30 (2017).

8. Kumar, K.A., Reddy, J.V.R., Sugunamma, V., and Sandeep, N. "Magnetohydrodynamic CattaneoChristov flow past a cone and a wedge with variable heat source/sink", Alex. Engng. J., 57, pp. 435-443 (2018).

9. Babu, M.J., Sandeep, N., and Saleem, S. "Free convective MHD Cattaneo-Christov flow over three different geometries with thermophoresis and Brownian motion", Alex. Engng. J., 56, pp. 659-669 (2017).

10. Reddy, J.V.R., Sugunamma, V., and Sandeep, N. "Cross diffusion effects on MHD flow over three different geometries with Cattaneo-Christov heat flux", J. Mol. Liq., 223, pp. 1234-1241 (2016).

11. Zhao, J., Zheng, L., Chen, X., and Zhang, X. "Unsteady Marangoni convection heat transfer of fractional Maxwell fluid with Cattaneo heat flux", Appl. Math. Mod., 44, pp. 497-507 (2017).

12. Sui, J., Zheng, L., and Zhang, X. "Boundary layer heat and mass transfer with Cattaneo-Christov doublediffusion in upper-convected Maxwell nanofluid past a stretching sheet with slip velocity", Int. J. Therm. Sci., 104, pp. 461-468 (2016).

13. Li, J., Zheng, L., and Liu, L. "MHD viscoelastic flow and heat transfer over a vertical stretching sheet with Cattaneo-Christov heat flux effects", J. Mol. Liquids, 221, pp. 19-25 (2016).

14. Liao, S.J. "Notes on the homotopy analysis method: Some definitions and theorems", Commun. Nonlinear. Sci. Numer. Simulat., 14, pp. 983-997 (2009).

15. Rashidi, M.M., Pour, S.A.M., Hayat, T., and Obaidat, S. "Analytic approximate solutions for steady flow over a rotating disk in porous medium with heat transfer by homotopy analysis method", Comp. Fluids, 54, pp. 1-9 (2012).

16. Rashidi, M.M., Feridoonimehr, N., Hosseini, A., Beg, O.A., and Hung, T.K. "Homotpy simulations of nanofluid dynamics from a non-linearly stretching isothermal permeable sheet with transpiration", $\mathrm{Mec}$ canica, 49, pp. 469-482 (2014).

17. Hayat, T., Hussain, Z., Farooq, M., and Alsaedi, A. "Effects of homogeneous and heterogeneous reactions and melting heat in the viscoelastic fluid flow", J. Mol. Liq., 215, pp. 749-755 (2016).

18. Khan, M. and Malik, R. "Forced convective heat transfer to Sisko nanofluid past a stretching cylinder in the presence of variable thermal conductivity", $J$. Mol. Liquids, 218, pp. 1-7 (2016).

19. Ganji, D.D., Fakour, M., Vahabzadeh, M., and Kachapi, S.H.H. "Accuracy of VIM, HPM and ADM in solving nonlinear equations for the steady threedimensional flow of a Walter's B fluid in vertical channel", Walailak J. Sci. Tech., 11, pp. 593-609 (2014).

20. Sheikholeslami, M., Ashorynejad, H.R., Domairry, G., and Hashim, I. "Flow and heat transfer of Cu-water nanofluid between a stretching sheet and a porous surface in a rotating system", Hindawi Publ. Corp. J. Appl. Math., 2012, Article ID 421320 (2012).

21. Ganji, D.D., Abbasi, M., Rahimi, J., Gholami, M., and Rahimipetroudi, I. "On the MHD squeezing flow between two parallel disks with suction or injection via HAM and HPM", Fron. Mech. Engng., 9, pp. 270-280 (2014).

22. Awais, M., Hayat, T., Nawaz, M., and Alsaedi, A. "Newtonian heating, thermal diffusion and diffusion thermo effects in an axisymmetric flow of Jeffery fluid with convective conditions", Bra. J. Che. Engng., 32, pp. 555-561 (2015).

23. Awais, M., Hayat, T., Alsaedi, A., and Asghar, S. "Time-dependent three-dimensional boundary layer flow of a Maxwell fluid", Comp. Fluids, 91, pp. 21-27 (2014).

24. Awais, M., Hayat, T., Irum, S., and Alsaedi, A. "Heat generation/absorption effects in a boundary layer stretched flow of Maxwell nanofluid: Analytic and numeric solutions", PLOS ONE, Article ID e0129814 (2015).

25. Saleem, S., Nadeem, S., and Awais, M. "Timedependent second-order viscoelastic fluid flow on rotating cone with heat generation and chemical reaction", J. Aerospace Engng., 29(4), Article No. 04016009 (2016).

26. Sadeghy, K., Hajibeygi, H., and Taghavi, S.M. "Stagnation-point flow of upper-convected Maxwell fluids", Int. J. Non-Linear Mechanics, 41, pp. 12421247 (2006).

27. Mukhopadhyay, S. "Heat transfer analysis of the unsteady flow of a Maxwell fluid over a stretching surface in the presence of a heat source/sink", Chinese Physics Letters, 29, p. 054703 (2012)

28. Hayat, T., Ali, S., Awais, M., and Obaidat, S. "Stagnation point flow of Burgers' fluid over a stretching surface", Progress in Computational Fluid Dynamics, 13, pp. 48-53 (2013).

\section{Biographies}

Aqsa is a PhD scholar in the Department of Mathematics, Quaid-i-Azam University, Islamabad, Pakistan. She obtained MSc degree from COMSATS Institute of Information Technology, Attock. Her research work is related to the numerical and analytical solutions to the nonlinear problems in the field of fluid dynamics.

Muhammad Yousaf Malik is currently working as the Chairman of the Department of Mathematics, Quaid-i-Azam University, Islamabad. He is a member 
of several national and international societies. His research interests are in numerical analysis, differential equations, and fluid dynamics. He has so far supervised several $\mathrm{PhD}$ and MPhil students.

Amna Imtiaz has received her MSc degree in Mathematics from COMSATS Institute of Information Technology, Attock. She is a junior research scholar currently working in the field of fluid mechanics. Her research interests are in mathematical modelling and the boundary layer flows in Newtonian and non-
Newtonian fluids.

Muhammad Awais has been working as Assistant Professor in the Department of Mathematics, COMSATS Institute of Information Technology, Attock, since 2013. His research interests are Newtonian and non-Newtonian fluid flows, biomechanics, and nanofluid rheology. He has several publications and has so far supervised $11 \mathrm{MSc}$ students as well. He is also member of several national and international research groups. 